\title{
Pain Management in an Adult Sickle Cell Infusion Center: A Quality Improvement Project
}

\author{
Lakesha Thomas ${ }^{\mathrm{a}}$, Geneva Caldwell ${ }^{\mathrm{b}}$
}

The purpose of this quality improvement project was to change the treatment process for patients during acute sickle cell vasoocclusive crisis with the intent of positively influencing patients' perceptions of pain management with medication therapy and improving patient satisfaction with the care they received. A structured survey using a likert scale regarding demographic data and patient satisfaction with treatment protocols, wait times, and clinic staff was obtained from 30 participants from the sickle cell clinic. An overall $63 \%$ (n-19) satisfaction rating among patients with the sickle cell infusion clinic revised process in comparison to the old process of presenting to the emergency room for treatment. The mean door to first dose of parenteral analgesic time was 73 minutes in the new process. The change in the process for admission/treatment positively influence their perception of managing their pain during vaso-occlusive crisis. The change in the process for admission/treatment positively influence their perception of managing their pain during vaso-occlusive crisis.

Keywords: sickle cell, sickle cell treatment, sickle cell disease, emergency room, chronic pain, infusion centers

\section{Problem Description}

Sickle cell disease (SCD) is a chronic condition that affects hundreds of thousands of people worldwide (O'Connor 2014). SCD is the most common inherited blood disorder in the United States of America affecting about 80,000 people (O'Connor 2014). As a result of this disease, patients endure frequent hospitalizations. The World Health Organization (WHO) recognizes sickle cell anemia as a priority for public health, especially because of problems with access to health services in several regions of the world (Cordeiro et al. 2014).

The sickle cell center in Baton Rouge, Louisiana, was implemented on July 1, 2013. The clinic also provides outpatient infusion services for treatment during acute vasoocclusive crisis, so that the patients do not have to utilize the hospital emergency room (ER). The median time from arrival to first dose of analgesia ranges from 1 hour and 17 minutes to 1 hour and 47 minutes in the emergency department (Whiteman et al. 2015). The clinic and infusion center patients are managed using a standardized treatment protocol. Since implementation of the sickle cell clinic, fiscal/operational data was gathered, revealing that emergency room utilization had decreased by $38 \%-45 \%$. Length of stay in the hospital had decreased by approximately two days over the past three years. Sickle cell patients in an outpatient day clinic are treated for acute vaso-occlusive episodes to avoid hospital admissions. These outcomes are in alignment with the financial outcomes of the institution; however, there is very little data to measure patient satisfaction and efficiency on the service. Patients expressed multiple complaints to the sickle cell clinic staff. As an intervention, an informal survey using a Likert scale was conducted among 30\% of the staff using the following questions: (1) Are patients in acute vaso-occlusive episodes evaluated within 30 minutes of presentation? (2) Is there adequate space available to treat patients who are in in acute vaso-occlusive episodes? There was a $100 \%$ consensus that patients are not being evaluated within 30 minutes of presentation, and a $100 \%$ consensus that there was not adequate space dedicated to the infusion clinic.

A second informal survey was conducted among $10 \%$ of clinic patients using the following questions: (1) Are you satisfied with the amount of time you waited to receive treatment? (2) Are you satisfied with the care that you received in the sickle cell infusion clinic? There was a $100 \%$ consensus among the surveyed patients that they were not satisfied with the infusion clinic wait times and the care received during treatment. After reviewing data from patients and staff, there proved to be a need to further explore patient perceptions of pain management, efficiency of services, and patient satisfaction in the sickle cell infusion clinic.

After reviewing current data from both patients and staff, a need surfaced to formally explore patient perceptions of pain management, efficiency of care, and equity of services within the local sickle cell infusion clinic. The question remained: Will a standardized process for treating pain related to vasoocclusive episodes improve patient satisfaction ratings for the clinic?

\section{Available Knowledge}

A systematic review of literature was conducted regarding sickle cell treatment and pain management in which three constant themes emerged: Barriers to care, provider perceptions and communication, and pain management interventions. Negative attitudes by providers and medical staff as well as external stressors (family concerns, financial issues, maintaining employment) can serve as barriers to care. A study by Anderson et al. (2014) revealed that negative attitudes of emergency department providers towards patients with SCD and pain management may cause treatment delays by providers when patients present with complaints of acute vaso-occlusive episodes. These delays in treatment can cause patients to have negative experiences with the emergency department which lead to a mistrust of medical providers and delays seeking treatment for future acute vaso-occlusive episodes. O'Connor et al. (2014) assessed some of the external stressors, challenges, and perceptions amongst people diagnosed with SCD. The most commonly identified stressors were family concerns, financial issues, frustration of being a caregiver to others when not feeling well, maintaining employment while sick, and negative attitudes of health care providers when seeking treatment. These stressors also contributed to delays in seeking 
treatment and noncompliance with prescribed treatments. Good provider relationships are integral to the development of trust and caring for adults with SCD.

The available research findings support patient perceived negative attitudes from healthcare professionals, discrimination, pain management protocols, and delivery of care times impact health outcomes in adults with SCD. The physiological and psychological effects of SCD create stressors that patients must deal with throughout their lifetime (Lucchesi et al. 2016). The studies included in the literature review revealed that patient perceptions have a significant effect on their relationships with healthcare providers for years. Negative attitudes and discriminatory behaviors are associated with decreased trust, non-adherence to physician recommendations and negative health outcomes (Haywood et al. 2010). These barriers can also cause delays and/or ineffective in the emergency department because of suspicions of drug addiction and stigma associated with the disease (Lucchesi et al. 2016). Expedient treatment with parenteral narcotic analgesia has been associated with decreased admissions and optimal outcomes. Implementation of outpatient infusion clinics dedicated to SCD decrease emergency department utilization, increase patient satisfaction by giving them the perception that their needs are being met in an expedient timeframe. Although the literature supports the dedicated infusion clinic model, there are gaps in the literature concerning patient's perceptions of pain management in sickle cell outpatient infusion centers (Whiteman el al 2015).

\section{Rationale}

Jean Watson's Theory of Human Caring (2008) and the FOCUS: PDCA methodology provided a relatable framework for this project with the aim to determine a process in which patients are treated in the Sickle Cell Infusion Center, improve efficiency of care, and reduce admissions secondary to vasoocclusive episodes. The overarching process for the acronym FOCUS is to find, organize, clarify, understand, and select a solution. PDCA, which is plan, do, check, act is used to evaluate the efficacy of the process and establish new policies moving forward. Investigation of patients' perceptions of pain management during vaso-occlusive episodes initiates the caritas process. The transpersonal relationship develops between the researcher, nurse, and patient when reports of uncontrolled pain from sickle cell disease are accepted as authentic and not the result of drug addiction or manipulation. Accepting the patient at their worse or while they are reacting to pain creates the caring occasion which has a substantial positive impact on the nurse-patient trust relationship. Trust is a major component of care and compliance in the treatment of SCD. Patients who mistrust providers and staff are less likely to be compliant and remain in care (Haywood, 2010). An inservice was held by the researcher to educate the staff on Watson's Theory of Caring and a post test was given to evaluate the staff's knowledge following the inservice.

The Focus PDCA methodology is used to guide the steps of the process change with the intent to maintain focus on why it should be changed and demonstrates accountability (Harris Roussel, \& Thomas, 2016). Before any piece of the process is changed, it requires a basic understanding of the problem.

Watson and FOCUS PDCA effectively guided the project to produce some positive changes in the process and patient perceptions of care. The intervention was expected to work because of literature showed that patients who trusted their providers and had dedicated centers for treatment showed increased compliance and better outcomes.

\section{Specific aims}

The purpose of this project was to determine if sickle cell patients who are receiving care for vaso-occlusive episodes in an infusion clinic along with oncology patients are satisfied with pain management services and if the patients felt that they were treated with respect. In addition, a goal of this quality improvement project is to improve the efficiency of healthcare services and enhance the quality of pain management for these patients at the time of crisis.

\section{Method}

The purpose of this project was to improve the process for treating sickle cell patients during an acute vaso-occlusive episode and improve their satisfaction rating for pain management over a short time frame.

\section{Context and Intervention}

The intervention was administered at a sickle cell outpatient infusion clinic located at an academic medical center in Baton Rouge. Prior to the implementation of this project, there were no standardized protocols for treatment of patients in acute vaso-occlusive episodes. There were also no standardized times to maximize efficiency of service. This clinic had no established timeframes for treatment from arrival to first dose of narcotic intravenous pain medication (door to treatment) time. There is only one dedicated full time employee for this operation and patients are managed on an unofficial "as soon as possible" basis upon arrival.

The outpatient infusion clinic was located approximately 1.2 miles from the location of the sickle cell infusion clinic on a high-traffic, major thoroughfare. With this design, patients were waiting up to 3 hours between presentation and treatment with analgesia. Frequently transportation was a problem for many patients. Patients expressed that this was inconvenient when receiving treatment. This information validated the need to systemically implement a revised patient flow process and capture their perception of pain management. Therefore the question remains: Will a standardized process for treating pain related to vaso-occlusive episodes improve patient satisfaction ratings for the clinic?

Eligible participants were patients enrolled in the sickle cell clinic at least age 18 years. The participants for this intervention were selected by a convenience sampling of patients presenting for treatment of acute vaso-occlusive episodes at the infusion clinic where the quality improvement project was conducted. Based on assessments of interventions used by previous researchers including surveys and personal interviews, three instruments were utilized to create the intervention which proved to directly capture and impact outcomes. They were:

Demographic Data Form. The demographic form collected nominal data including age, gender, the amount of time enrolled in the sickle cell clinic and infusion clinic, and the number of acute vaso-occlusive episodes over 6 months. 
Research Article (2019)

Table 1.1: Demographics

\begin{tabular}{|lr|r|rr|rr|}
\hline $\begin{array}{l}\text { Demographic } \\
\text { Variable }\end{array}$ & $\begin{array}{r}\text { Sample } \\
\text { Size }\end{array}$ & Mean & Median & $\begin{array}{r}\text { Standard } \\
\text { Deviation }\end{array}$ & Minimum & Maximum \\
Age & 30 & 28.2000 & 27.0 & 7.7076 & 18.0 & 52 \\
\hline Years & 30 & 2.1767 & 2.0 & 1.2167 & 0.4 & 4 \\
\hline Episodes & 30 & 4.4000 & 4.0 & 1.9405 & 1.0 & 10 \\
Time & 30 & 73.1333 & 72.5 & 10.2780 & 58.0 & 94 \\
\hline
\end{tabular}

Table 1.2: Gender Distribution

\begin{tabular}{|l|l|l|}
\hline Gender & Frequency & Percent \\
\hline Male & 14 & 46.67 \\
\hline Female & 16 & 53.33 \\
\hline
\end{tabular}

Interview Form. Patient perception data was collected using a structured written survey. This questionnaire was designed to assess patients' perceptions of the treatment and efficiency of healthcare services provided in the clinic. The written patient satisfaction survey consists of nine questions related to providers, staff, treatment times, and level of satisfaction. The question ratings were based on the Likert scale. Response options to the survey questions were: "not at all," "a little bit," "somewhat," "quite a bit," and "very much."

NRS-11. The Numeric Pain Rating Scale (NRS-11) is a onedimensional model measure of pain intensity in adults, including those with chronic pain due to sickle cell disease (Figure 1). This pain-rating scale was developed by MaCaffery and Beebe (1989) to track small changes in pain without discrimination by using numerical values as perceived by the patient. The 11-point numeric scale ranges from " 0 " representing one pain extreme ("no pain") to "10" representing the other pain extreme ("pain as bad as you can imagine" or "worst pain imaginable").

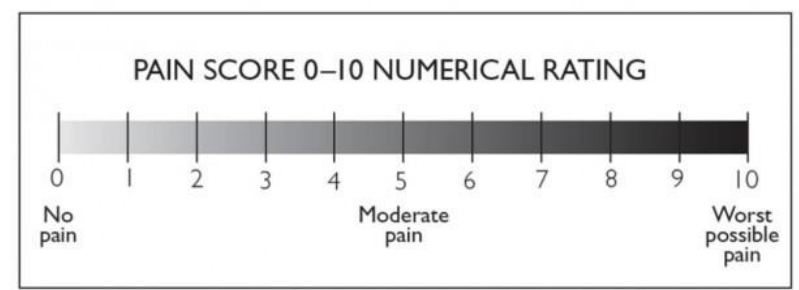

Figure 1: NRS-11 Pain Rating Scale

\section{Measures}

The researcher met with staff prior to implementation date to provide information on the process and goals of this quality improvement project. All questions and concerns from staff were addressed. A convenience sample of participants was identified as patients presented to the clinic with a vasoocclusive episode. The researcher approached these patients prior to treatment in the infusion clinic and asked if they were willing to participate in the intervention. If patients agreed to participate, the project was explained in extensive detail and a written informed consent was obtained. Potential risks and benefits were explained verbally and in writing to each participant. The RN admitted the patients to the infusion clinic and a pre-treatment pain score was assessed using the NRS- 11 . Intravenous access was secured, and intravenous hydration therapy was initiated. A dose of either morphine or hydromorphone was administered to the patients by intravenous route. Using NRS-11, each patient's pain was reassessed one hour after receiving IV analgesia. Prior to discharge from the infusion clinic, the patients completed the demographic form and structured questionnaire. The infusion clinic nurse collected the completed surveys from the patients to prevent the patients from experiencing any coercion by the researcher. The participants were given a debriefing form with contact information of the researcher in case they had any questions or concerns following the survey. All data collection forms were assigned a code. The master list of participants' names/codes and consent forms were kept separate from the demographic forms, patient satisfaction survey forms, and NRS-11 pain assessment forms. Completed instruments were recorded into an Excel spreadsheet on a password protected work laptop. Paper copies of surveys were locked in a filing cabinet in the researcher's office, and only the researcher has a key.

\section{Analysis}

The results of the data are separated into four sections: demographic information, patient satisfaction survey responses, decrease in pre- and post-treatment pain, and time between arrival and first dose of parenteral analgesia. The statistician analyzed the data using descriptive statistics, Pearson Correlation, one sample t-tests, and other appropriate analyses as deemed necessary. The results of the data analysis were interpreted and synthesized by the researcher.

\section{Outcome Analysis}

Data was collected using the structured surveys over an eight-week period from January 30, 2017, to March 31, 2017. The population surveyed consisted of 30 adults with sickle cell disease ranging from age 18 to 52. Among participants, the mean number of vaso-occlusive episodes experienced in a sixmonth period was 4.4. The mean amount of years the participants were enrolled in the sickle cell center was 2.17 years. The average length of time from arrival to first dose of parenteral narcotic analgesia was 73.13 minutes. The longest wait time among participants was 94 minutes and the shortest time was 58 minutes.

\section{Description of Data Results}

A frequency distribution was used to analyze the gender distribution of the project participants $(n=30)$. The purpose of a frequency distribution is to list all possible measures of a variable and tally each datum on the listing (Grove et al. 2016). Fourteen participants were males and sixteen (53.33\%) participants were females.

\section{Patient Satisfaction}

A frequency distribution was also used to analyze the results of the patient satisfaction survey (Table 2). The first question patients were designed to ascertain how patients 
Research Article (2019)

perceived the whether their complaints were treated as urgent by nurses and providers. Seventeen participants $(56.67 \%)$ perceived that their complaints were treated as urgent. Question \#2 ascertained how satisfied patients were with the wait time from arrival to first dose of pain medication. Eighteen participants $(60 \%)$ were satisfied with their wait time from arrival to first dose of pain medication. Question \#3 asked was to ascertain if the patients felt that the clinic providers and nurses listened and if they provided thorough answers to patient's questions and concerns. Twenty-one participants (70\%) were satisfied with attention providers and nurses gave their questions and concerns. Question \#4 asked if patients were satisfied with the frequency in which the nurses assessed their pain level during treatment. Twenty-one participants (70\%) were satisfied with the frequency of pain reassessment by the nurse. Question \#5 measured if treatment (i.e. intravenous fluids and intravenous pain medication) effectively relieved the patient's pain. Twelve participants (40\%) said the treatment satisfactorily relieved their pain. Question \#6 asked if the patient's pain had decreased from arrival in the infusion clinic to discharge. Seven participants $(23.33 \%)$ indicated that their pain decreased "very much;" however, twelve participants (40\%) felt their pain only decreased "quite a bit." Question \#7 was asked whether patients felt that the nurses and providers were truly concerned about their comfort level during treatment. Twenty-one participants $(70 \%)$ indicated that the nurses and providers were truly concerned about their level of comfort during treatment at the infusion clinic. Question \#8 asked if patients felt they were ready to be discharged following the treatment in the infusion clinic. Thirteen participants $(43.33 \%)$ felt that their pain was controlled enough to be discharged home following treatment. Question \#9 determined the patient's overall satisfaction with their treatment experience in the infusion clinic. Nineteen participants $(63.33 \%)$ were satisfied with their overall treatment experience in the infusion clinic.

Table 2: Individual Question Responses

\begin{tabular}{|c|c|c|c|c|c|c|c|c|c|c|}
\hline \multirow{2}{*}{$\begin{array}{l}\text { Question } \\
\text { Urgent Complaints }\end{array}$} & \multicolumn{2}{|c|}{$\begin{array}{l}\text { Not at all } \\
\text { Freq. } \%\end{array}$} & \multicolumn{2}{|c|}{$\begin{array}{l}\text { A little bit } \\
\text { Freq. \% }\end{array}$} & \multicolumn{2}{|c|}{$\begin{array}{l}\text { Somewhat } \\
\text { Freq. \% }\end{array}$} & \multicolumn{2}{|c|}{$\begin{array}{l}\text { Quite a Bit } \\
\text { Freq. \% }\end{array}$} & \multicolumn{2}{|c|}{$\begin{array}{l}\text { Very much } \\
\text { Freq. } \%\end{array}$} \\
\hline & & & 2 & $6.67 \%$ & 5 & $16.67 \%$ & 6 & $20.00 \%$ & 17 & $56.67 \%$ \\
\hline Wait time & & & 3 & $10.00 \%$ & 1 & $3.33 \%$ & 8 & $26.67 \%$ & 18 & $60.00 \%$ \\
\hline Provider Satisfaction & 1 & $3.33 \%$ & 2 & $6.67 \%$ & 2 & $6.67 \%$ & 4 & $13.33 \%$ & 21 & $70.00 \%$ \\
\hline Pain reassessment & 1 & $3.33 \%$ & & & 3 & $10.00 \%$ & 5 & $16.67 \%$ & 21 & $70.00 \%$ \\
\hline Pain management & & & 2 & $6.67 \%$ & 6 & $20.00 \%$ & 10 & $3.33 \%$ & 12 & $40.00 \%$ \\
\hline Pain relief & & & 5 & $16.67 \%$ & 6 & $20.00 \%$ & 12 & $40.00 \%$ & 7 & $23.33 \%$ \\
\hline Provider concern & & & & & 2 & $6.67 \%$ & 7 & $23.33 \%$ & 21 & $70.00 \%$ \\
\hline Discharge readiness & 1 & $3.33 \%$ & 1 & $3.33 \%$ & 6 & $20.00 \%$ & 9 & $30.00 \%$ & 13 & $43.33 \%$ \\
\hline Overall satisfaction & & & & & 4 & $13.33 \%$ & 7 & $23.33 \%$ & 19 & $63.33 \%$ \\
\hline
\end{tabular}

\section{Pain}

A one sample t-test was used to determine if changes in pain were statistically significant between pretreatment and post-treatment values. The purpose of the t-test was to determine significant differences between measures of two samples (Grove et al., 2016). The null hypothesis found no change in pain between pre-treatment and post-treatment pain scores (Table 3). The average participant recorded a 5-point drop in pain between pre-treatment and post treatment within the infusion clinic rejecting the null hypothesis with a ( $p$ $<0.0001)$
Table 3.0: Difference in Pre and Post Treatment Value

\begin{tabular}{|ll|l|l|l|l|l|l|}
\hline$N$ & Mean & Median & Std. Dev & Min & Max & TSTAT & P-Value \\
\hline 30 & -5.33333 & -5 & 2.29442 & -10 & 0 & -12.7317 & $<0.0001$ \\
& & & & & & & \\
\hline
\end{tabular}

A Pearson Correlation Coefficient was used to determine if there was a connection between decreased pain after treatment and patient satisfaction total score. A Pearson Correlation Coefficient is used to determine the relationship between two variables (Grove et al., 2016). The results validated the null hypothesis that there was not a statistically significant correlation between pain relief and satisfaction with healthcare services at the sickle cell infusion clinic ( $p=0.3030)$ (Table 4).

Table 4: Changes in Pain vs Patient Satisfaction

\begin{tabular}{|l|l|l|l|lc|}
\hline \multicolumn{1}{|c|}{ Variable } & N & Mean & Std Dev & Correlation & p-value \\
\hline $\begin{array}{l}\text { Change (Post Medication - } \\
\text { Pre Medication) in Pain }\end{array}$ & 30 & -5.33333 & 2.29442 & -0.19451 & 0.3030 \\
\hline $\begin{array}{l}\text { Patient Satisfaction Total } \\
\text { Score }\end{array}$ & 30 & 38.50000 & 5.02236 & & \\
\hline
\end{tabular}

A one-way ANOVA was used to determine the relationship between patients who reported being "somewhat," "quite a bit," and "very much" overall satisfied with the infusion clinic treatment, quality of healthcare services, and decrease in pain levels. The purpose of an ANOVA is to examine difference among two or more group by comparing the variability between the groups with the variability within the groups (Grove et al, 2016). The null hypothesis for this analysis is revealed no difference in pain relief among the three patient satisfaction groups ("somewhat," "quite a bit," and "very much"). When the three different groups were compared, an Ftest revealed that those who rated their overall experience as "somewhat satisfied" had a smaller decrease in pain than those who rated their experience "quite a bit" or "very much" satisfying ( $p=0.0014)$ (Table 5).

Table 5: Pain Difference by Overall Satisfaction

\begin{tabular}{|l|c|r|r|r|c|}
\hline \multicolumn{1}{|c|}{ Satisfaction } & N & Mean & Std Dev. & F Stat & P - value \\
\cline { 1 - 3 } Somewhat & 4 & -2.50 & 2.08 & \multirow{2}{*}{8.52} & 0.0014 \\
\cline { 1 - 4 } Quite a bit & 7 & -7.29 & 2.14 & & \\
\cline { 1 - 4 } Very much & 19 & -5.21 & 1.72 & & \\
\hline
\end{tabular}

Data was also collected comparing overall satisfaction with the infusion clinic from arrival to first dose of parenteral analgesia. Collecting this time variable informationallowed the researcher to determine if wait times were associated with the perception of patient experience. The analysis revealed no statistically significant correlation between first dose of analgesia time and patient satisfaction ( $p=0.7429$ ) (Table 6). Participants reported overall satisfaction with the new sickle cell infusion clinic process. Although the arrival-to-first-dose of narcotic analgesia time was 73 minutes, participants who were more satisfied with 
Research Article (2019)

care which included theory of human care had better response to treatment than those unsatisfied with care.

Table 6: Time From Arrival to First Dose: Overall Satisfaction

\begin{tabular}{|c|c|c|c|c|c|}
\hline Satisfaction & $\mathbf{N}$ & Mean & Std. Dev & F - Stat & P-Value \\
\hline Somewhat & 4 & 75.25 & 12.20 & 0.30 & \multirow[t]{3}{*}{0.7429} \\
\hline Quite a bit & 7 & 75.00 & 13.47 & & \\
\hline Very much & 19 & 72.00 & 9.01 & & \\
\hline
\end{tabular}

\section{Ethical Considerations}

This project was submitted to and approved by the University Institutional Review Board and the hospital's evidence-based practice committee. The project was also submitted to the Medical Director of the Sickle Cell Clinic. After approval was granted by all three entities, the project was initiated on January 30, 2017. Special consideration was made to provide an informed consent form detailing the goals, risks, and benefits of the project which were also verbally explained in extensive detail to participants. The researcher was the only person with access to the data during and after the project. After five years, all information will be destroyed via shredding and destruction of flash drive. There will be no monetary costs or gains for participating in the project.

\section{Discussion}

The purpose of this project was to determine patient priorities and satisfaction when coming into the infusion center for acute vaso-occlusive crisis management. Patients were currently being treated for vaso-occlusive crises in a clinic that also provided care to an oncology patient population. This issue impeded the timeframe in which SCD patients could be treated because there was only one nurse dedicated to caring for all the sickle cell patients in the infusion center. The results of the project revealed overall patient satisfaction with the sickle cell infusion clinic and satisfaction with the knowledge and level of care given by the providers and staff. According to the data, SCD patients perceived that the process of the sickle cell infusion clinic made was more efficient than the previous process of presenting to the emergency room for treatment. This overall level of satisfaction corroborates the literature as stated by Whiteman et al (2015) that patients feel like they receive excellent care when treated in a dedicated sickle cell infusion clinic. Jean Watson's Theory of Human Caring concepts (2008) were actualized in this situation. Once the researcher addressed the issue of dissatisfaction with care and rearranged the intake process to make the patient experience more efficient, the nurse and patient came together and interacted in such a way that an occasion for human caring was created. The caring healing modalities occurred when the researcher was able to go beyond the specific patient interaction but altered the environment and flow process to accommodate the patient in a caring way. The FOCUS-PDCA process improvement guided the flow of service and treatment in an organized and methodical manner. All treatments were a cascade of the previous steps in the process, which made it easy to execute the overall plan.

\section{Summary}

Administrative leaders are constantly looking for ways to improve their healthcare services and need hard data to support the decisions related to those activities. The sickle cell infusion clinic provides an alternative approach in the treatment of vasoocclusive episodes. Providing these services in this clinic has demonstrated a great financial outcome in saving costs of hospitalization in the sum of $\$ 3$ million dollars over a 2-year time span. The findings from this quality improvement project strongly recommend that systemic change that includes Watson theory of caring and FOCUS-PDCA is needed in order to improve patient care satisfaction ratings and overall clinical outcomes. Although the patients were satisfied with their care, these efforts should be made to improve the overall door-todose time which is 30 minutes (Whiteman et al, 2016). As patients continue to experience positive outcomes, they will spread the word to other patients, and this will increase number of visits and revenue.

\section{Interpretation}

Results from this project revealed opportunity for improvement and gaps in providing quality health care services to SCD patients. The average door-to-first-dose time was 73 minutes, which exceeds the American Pain Society's recommendation of 30 minutes for best practice outcomes. One reason for this delay in treatment is because the location of the infusion clinic from where assessments are provided by the providers prior to receiving treatment in the infusion clinic. Coding restrictions and office visits prior to treatment caused delays and constraints to efficient treatment. In this incident, patients wait prior to being taken to exam rooms to be evaluated by provider and then they have to wait again upon presenting to the infusion clinic following the provider visit. Another factor causing a delay in care is the use of one full-time employee in the infusion clinic. The infusion clinic is staffed with only one registered nurse who is responsible for providing total care for up to six patients simultaneously. This creates delays in retrieving patients from the waiting area and administration of medication depending on the volume of patients. This project was the first to evaluate patient perceptions of this program since opening in 2014. This data provides compelling evidence to help make improvements for the infusion clinic process, such as the addition of a full-time employee in the infusion clinic or the possibility of moving infusion to another area where office visits can be billed in that space as opposed to the current protocol.

The clinical significance of these findings help: (1) develop a refined process for acute care management that will ensure that all patients receive safe, effective, efficient, and equitable care; (2) add full-time employees that resonate with the workload factor for the increase in patients seen in the clinic; and (3) improve the timeliness of pain control in a patient-centered approach to improve patient satisfaction outcomes.

\section{Impact on the Population}

The interventions utilized in this project are not only aimed at improving clinical outcomes for SCD patients but also saving money for hospitals. Hospital administrators can also facilitate interdepartmental collaboration between the inpatient 
hematology/ oncology unit, emergency department, infusion centers, and case management to help meet patient goals. Another set of stakeholders would be healthcare providers in primary care, hematology, and emergency departments. According to Lanzkron et al. (2015), the cost for sickle cell treatment in the emergency department without admission is $\$ 1,975$ per visit compared to $\$ 739$ per visit in the sickle cell infusion clinic. The treatment cost in the infusion clinic provides a significant savings especially in treatment for high utilizers. The sickle cell infusion clinic offers an alternative to the emergency departments which decreases wait times for patients and door-to-first-dose times on pain medications; therefore, relieving pressure on the emergency room as well as placing the hematologist and primary care providers in control of the patient's course.

Clinicians will benefit from this project because the evidence collected provides insight into the patients' perspectives on how their pain is being managed. This project will also provide data that can be used by clinicians to help improve delivery of care for SCD patients. Also, evaluations of the patients' perspectives of pain management lead to quality improvement processes to streamline care and equity in the infusion clinic.

Primary care providers and hematologists have the most amount of interaction with these patients. The actions of these providers directly affect the health outcomes. Patients with SCD are the most important stakeholders because the outcomes drastically impact their quality of life.

\section{Limitations}

There were several limitations and barriers presented during this project. First, the sample size is small, so the results are not representative of a large sickle cell patient population. Second, the project was conducted at one clinic site therefore limiting generalizability. Third, the data collected was selfreported by the participants, therefore satisfaction or pain score could have been over-exaggerated or minimized. Patients receiving infusions are all located in one room; therefore, they were able to discuss the questions on the survey among themselves, and their responses may have been the result of pressure from their peers. Fourth, the time constraints to participate in this project may be perceived as another delay in care. Fifth, data regarding the type of SCD each participant was not collected. The type of SCD affects the intensity and severity of the vaso-occlusive episode and collecting this information could have determined higher satisfaction in services and equity. Despite these limitations, the data collected is valuable to process improvement and enhance quality of services. Limitations of outcomes for this project apply to this clinic specifically. This may not provide a generalization for all other clinics where sickle cell patients are treated for vaso-occlusive crisis. However, the strategies in this project may be effectively transitioned to other settings. The findings are subjective and limited to the patients included in this project which may or may not be reliable. Patients may not be truthful or may have withheld information for fear of not receiving appropriate care. The expectation and assumption is that the patients provided accurate and reliable information, the patients completed all aspects of this project, and the clinic agency continued to support this project until completion. The researcher also assumed the data obtained from this project will be taken into consideration and implemented by the facility.

\section{Conclusions \\ Future Implications for Practice}

The findings of this quality improvement project indicate that patients tend to be more satisfied with treatment when they perceive that their needs are being addressed in an expedient manner with open accessibility. This information can be used to provide education for providers concerning perceptions of SCD patients when the treatment of vaso-occlusive episodes is addressed immediately upon presentation to the clinic or emergency department. Also, processes and practices to accommodate SCD patients when experiencing acute vasoocclusive crisis episodes should be altered in order to provide immediate relief and facilitate improved outcomes with deliberate use of Jean Watson's Theory of Human Caring. Emergency department providers should be further educated on the infusion clinic's outpatient treatment approaches to avoid admissions which can be efficiently served within infusion clinics. Future research should be focused on the expansion of this quality improvement project to include a comparison of treatment in the emergency department and treatment in the infusion clinic. This advanced research should also include a comparison of sickle cell clinic and emergency department admission rates, arrival-to-dose times, and patient ratings of care received. Formal research would provide more information on both departments and their protocols and be used to revise protocols as needed.

\section{References}

Anderson, N., Bellot, J., Oke, O., Ballas, S. (2014). Characteristics of Acute Care Utilization of a Delaware Adult Sickle Cell Disease Patient Population. Population Health Management, 17(1), 60-65.

Centers for Disease Control and Prevention. (2017). Preventing Infections in Cancer Patients. Retrieved on May 11, 2017 from https://www.cdc.gov/cancer/dcpc/resources/features /preventinfections/

Coleman, B., Caird, H., McGowan, J., Benjamin, M. (2016). How sickle cell disease patients experience, understand and explain their pain: An interpretative phenomenological analysis study. British Journal of Health Psychology. 21: 190- 203.

Ezenwa, M., Molokie, R., Wilkie, D., Suarez, M., Yao, Y. (2015). Pain Management Nursing, 16(3), 294-306.

Harris, J., Roussel, L., Dearman, C., \& Thomas, P. (2016). ( $2^{\text {nd }}$ ed.) Project planning and Management: A guide for nurses and interprofessional teams. Burlington, MA, Jones \& Bartlett.

Haywood, C., Lanzkron, S., Ratanawonsga, N., Bediako S., Lattimer, L., Neil, Powe, Beach, M. (2010). The Association of Provider Communication with trust among adults with sickle cell disease. Journal of General Internal Medicine, 25(6), 543-548.

Lanzkron, S., Carroll, P. C., Hill, P., David, M., Paul, N., \& 
Haywood, C. (2015). Impact of a Dedicated Infusion Clinic for Acute Management of Adults with Sickle Cell Pain Crisis. American Journal of Hematology, 90(5), 376-380.

Lotich, P. (2014). FOCUS and PDCA Improvement Methodologies. Smart Church Management. Retrieved from https://smartchurchmanagement.com/what-is-focuspdca-

Lucchesi, F., Figueiredo, M., Mastandrea, E., Levenson, J., Smith, W., Jacinto, A., Citero, V. (2016). Physicians' Perception of Sickle-Cell Disease Pain. Journal of the National Medical Association, 108(2), 113- 118.

McCaffery, M. \& Beebe, A. (1989). Pain: Clinical manual for nursing practice. Mosby, St. Louis, MO.

Moran, K. (2017). Developing the Scholarly Project. In K. Moran. R. Burson, \& D. Conrad (Eds.) The Doctor of Nursing Practice Scholarly Project: A Framework for Success. ( $2^{\text {nd }}$ ed.). Burlington, MA, Jones \& Bartlett.
O'Connor, S., Hanes, D., Lindsey, A., Weiss, M., Petty, L., Overcash, J. (2014). Attitudes Among Healthcare Providers and Patients Diagnosed with Sickle Cell Disease: Frequent Hospitalizations and Stressors. Clinical Journal of Oncology Nursing, 18(6), 675680.

Tanabe, P., Hafner, J., Martinovich, Z., Artz, N. (2012). Adult emergency department patients with sickle cell pain crisis: results from a quality improvement learning collaborative model to improve analgesic management. Journal of the Society for Academic Emergency Medicine. 19(4), 430-438.

Whiteman, L. N. (2015). Quality Improvement Process in a Sickle Cell Infusion Center The American Journal of Medicine, 128(5). 541-544.

Grove, S. K, Gray, J. R., Sutherland S. (2016). The Practice of Nursing Research: Appraisal, Synthesis, and Generation of Evidence (8th ed). St. Louis, MO: Elsevier Saunders. 